\title{
A Herschel View of Dust Evolution in Protoplanetary Disks
}

\author{
Catherine Espaillat \\ Boston University \\ Department of Astronomy \\ 725 Commonwealth Avenue, Boston, MA 02215, USA \\ email: cce@bu.edu
}

\begin{abstract}
The details of how protoplanetary disks evolve from initially well-mixed distributions of gas and dust to systems composed mostly of rocky planets and gas giants like our own solar system is a fundamental question in astronomy. It is widely accepted that the first step in planet formation is dust grain growth and settling to the disk midplane. This dust evolution in disks can be studied in greater detail with far-infrared and submillimeter wavelength observations, which offer us unique access to the outer disk's deeper layers. Here we present Herschel far-infrared and submillimeter spectra of GM Aur taken with PACS and SPIRE. GM Aur is a transitional disk, whose inner disk hole is proposed to have been cleared by yet unseen planets. By utilizing Herschel data, we can potentially link the properties of dust evolution in the outer disk to dust clearing in the inner disk. In particular, preliminary SED modeling presented here suggests that GM Aur may have a lower gas-to-dust mass ratio than typically assumed for disks, which may be linked to disk clearing by planets. With further study, such Herschel data may provide insight for theoretical modeling of dust evolution and planet formation.
\end{abstract}

Keywords. (stars:) planetary systems: protoplanetary disks, stars: formation, accretion, accretion disks

\section{Introduction}

Many questions exist concerning how the gas and dust in a disk around a typical T Tauri star (TTS) evolves into a planetary system and observations of TTS may provide clues. Some objects in particular that have gained increasing attention in this regard are those dubbed "transitional disks" (Strom et al. 1989). Their spectral energy distributions (SEDs) lack excess emission above the stellar photosphere at infrared (IR) wavelengths and they were first identified using near-infrared (NIR) ground-based photometry and IRAS mid-IR (MIR) photometry Strom et al. 1989; Skrutskie et al. 1990). These "dips" were thought to indicate a hole in the dust distribution of the disk.

IR dips in the SEDs of several transitional disks were later confirmed with the Spitzer Space Telescope (Werner et al. 2004). The Infrared Spectrograph (IRS; Houck et al. 2004) provided unprecedented, simultaneous wavelength coverage between $\sim 5 \mu \mathrm{m}$ and $38 \mu \mathrm{m}$, uncovering new details regarding these IR dips (Furlan et al. 2006). Many transitional disk SEDs had nearly photospheric NIR $(1-5 \mu \mathrm{m})$ and MIR $(5-20 \mu \mathrm{m})$ emission, coupled with substantial emission at wavelengths beyond $\sim 20 \mu \mathrm{m}$.

Detailed modeling of many transitional disk SEDs incorporating Spitzer data has been performed. SEDs of transitional disks with little or no NIR and MIR emission have been fit with models of inwardly truncated optically thick disks (Calvet et al. 2002; Calvet et al. 2005; Espaillat et al. 2007; Espaillat et al. 2008). The inner edge or "wall" of the outer disk is frontally illuminated by the star, dominating most of the emission seen in the IRS spectrum (e.g., GM Aur; Figure 1, left). Some of the holes in transitional disks 

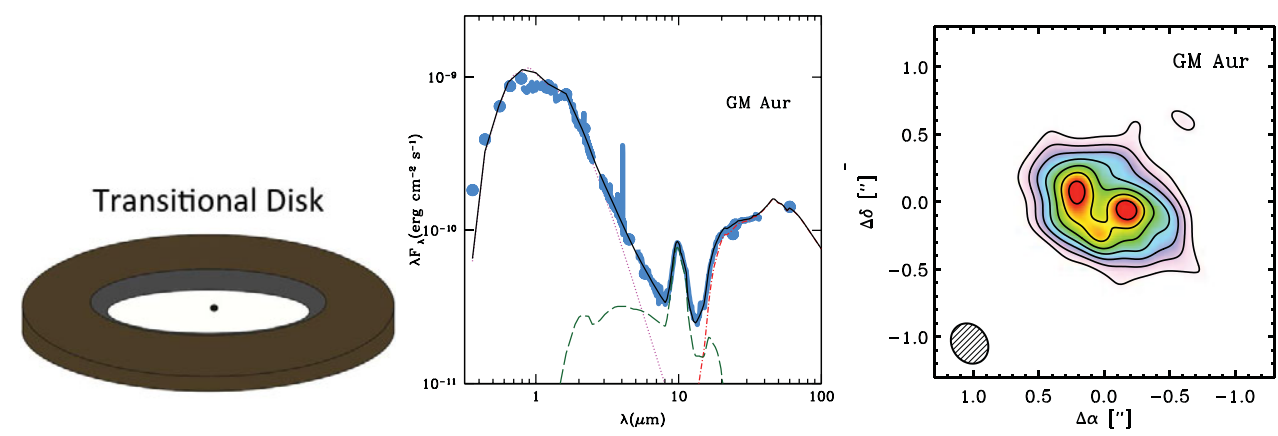

Figure 1. Left: Schematic of transitional disk structure. Progressing outward from the star (black) is the disk hole (white), outer wall (light gray), and outer disk (dark gray). Center: Observed (symbols) and simulated (black line) SED of the transitional disk of GM Aur taken from Espaillat et al. (2011). The transitional disk model consists of the stellar photosphere (dotted line) and an outer wall and outer disk (dot-short-dash line). Within the cleared dust region there is $\sim 10^{-11} \mathrm{M}_{\odot}$ of submicron-sized optically thin dust (long-dash line) which creates the strong $10 \mu \mathrm{m}$ silicate feature. Right: $345 \mathrm{GHz}(850 \mu \mathrm{m})$ submillimeter continuum image of GM Aur from Andrews et al. (2011), confirming the presence of a clearing with a $20 \mathrm{AU}$ radius.

are relatively dust-free (e.g., DM Tau; Calvet et al. 2005; Espaillat et al. 2010) while SED model fitting indicates that others with strong $10 \mu \mathrm{m}$ silicate emission have a small amount of optically thin dust in their disk holes to explain this feature (e.g., GM Aur; Figure 1, center; Calvet et al. 2005; Espaillat et al. 2010). For many transitional disks, the truncation of the outer disk has been confirmed with submillimeter and millimeter interferometric imaging (e.g., GM Aur; Figure 1, right; Hughes et al. 2007; Hughes et al. 2009; Andrews et al. 2009; Andrews et al. 2010; Andrews et al. 2011).

The distinct SEDs of transitional disks most likely signify that these objects are in an important phase of disk evolution. Many researchers have proposed that these disks are forming planets on the basis that cleared regions in disks are predicted by planet formation simulations (e.g., Paardekooper \& Mellema 2004; Zhu et al. 2012). To explore the potential link between transitional disks and planet formation, we present Herschel farinfrared and submillimeter spectra of GM Aur. Data at these wavelengths trace regions deeper in the disk, where dust evolution is expected to occur. By utilizing Herschel data of transitional disks, we can look for new evidence supporting the presence of planets in disks. In turn, the results can be used to inform models of dust evolution and planet formation.

\section{Overview}

The pre-Herschel SED of GM Aur (Figure 2, left) lacks substantial coverage in the far-infrared (FIR) and submillimeter wavelengths, even though this object is one of the best-studied in the literature from the U-band to millimeter wavelengths. Adding in Herschel PACS and SPIRE spectra significantly expands the wavelength coverage in the SED (Figure 2, right). Preliminary modeling analysis of the updated SED of GM Aur has yielded an interesting result. We find that the best-fitting model to the pre-Herschel SED of GM Aur, which was presented by Espaillat et al. (2011), does not fit the observed emission at $\sim 100-500 \mu \mathrm{m}$ (Figure 3 , left). Extra emission at these wavelengths is seen from the disk that the previous model does not predict.

In our new preliminary fit to the updated SED of GM Aur, we can account for the extra emission in the FIR and submillimeter with a disk that is more massive (Figure 3, 



Figure 2. Left: Observed SED of GM Aur taken from Espaillat et al. (2011). We show SpeX and IRS spectra from Espaillat et al. $(2010,2011)$ as well as the stellar photosphere (dotted line). Open circles correspond to ground-based (UBVRIL) photometry taken from Kenyon \& Hartmann (1995). Closed circles are 2MASS photometry from Skrutskie et al. (2006). Triangles are Spitzer photometry from Luhman et al. (2010). Squares correspond to IRAS and millimeter fluxes. IRAS data are taken from Weaver et al. (1992). Millimeter data were obtained from Andrews \& Williams (2005), Weintraub et al. (1989), Beckwith \& Sargent (1991), and Hughes et al. (2009). Right: Observed SED of GM Aur including preliminary Herschel PACS and SPIRE spectra. Herschel significantly expands our wavelength coverage at FIR and submillimeter wavelengths. Note that the processing of the Herschel data may be improved with future pipeline releases and we refer the reader to Espaillat et al. (in preparation) for a more recent data reduction and relevant details.
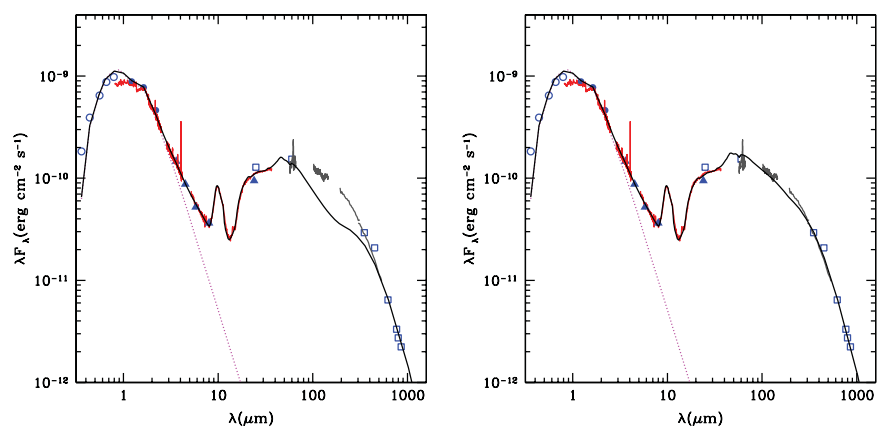

Figure 3. Left: Model SED (black line) presented in Figure 1 from Espaillat et al. (2011) overlaid on the updated SED of GM Aur from Figure 2 (right). The model is a good fit to the pre-Herschel SED presented in the left panel of Figure 2. It does not fit the updated SED at 100-500 $\mu \mathrm{m}$, however, highlighting the importance of including Herschel data in SED modeling. Right: Preliminary model fit (black line) to the updated SED of GM Aur. This is a better fit to the Herschel data at 100-500 $\mu \mathrm{m}$; more details can be found in Section 2. We stress that this is a preliminary fit and that analysis is still underway. We refer the reader to Espaillat et al. (in preparation) for the most recent SED modeling analysis results.

right). However, this disk is Toomre unstable. To reconcile this dilemma, GM Aur may have a lower gas-to-dust mass ratio in its disk than what is typically assumed (i.e., 100). A disk model with a lower gas-to-dust mass ratio can produce similar emission levels but with a less massive disk since $\mathrm{F}_{m m} \propto \mathrm{M}_{\text {dust }} \propto($ dust-to-gas ratio $) \times \mathrm{M}_{\text {gas }}$. Therefore, with a lower gas-to-dust ratio (i.e., a higher dust-to-gas ratio), a similar millimeter flux could be obtained with less gas (which is the bulk of the disk mass). We stress that this result is preliminary and other possibilities that may be more important need to be explored before this initial result is confirmed. We refer the reader to Espaillat et al. (in preparation) for more recent analysis results. 


\section{Implications}

Lower gas-to-dust mass ratios in disks have been proposed previously. One example is the transitional disk of TW Hya where Thi et al. (2010) inferred a gas-to-dust ratio of $\sim 30$ using PACS observations of the [OI] line. This result, however, has been recently disputed by Bergin et al. (2013) who find the gas-to-dust ratio of TW Hya is $~ 100$ using the HD line. More work needs to be done to decipher disk gas-to-dust mass ratios.

In the meantime, we can speculate that if indeed the gas-to-dust mass ratios of transitional disks is lower, this difference may be related to the mechanism that is potentially causing the holes in these disks, namely planets. Recently, there have been steps to more closely tie theory to observations by simulating disk clearing due to dust filtration. The dust filtration model predicts substantial holes in the large dust grain distribution of the disk, while the gas and small dust grain distribution have undetectable gaps (Zhu et al. 2012). This mechanism could potentially lead to lower gas-to-dust ratios in disks if indeed gas is being depleted in the outer disk as it filters through into the inner disk and is accreted onto the star while large dust grains are being held back in the outer disk. Still, dust filtration does not explain all aspects of transitional disk observations (e.g., MIR deficits), indicating that we are still missing important physics in these objects. More theoretical and observational work needs to be done and the particular case of GM Aur will be explored in more detail by Espaillat et al. (in preparation).

\section{References}

Andrews, S. M. \& Williams, J. P. 2005, ApJ, 631, 1134

Andrews, S. M., Wilner, D. J., Hughes, A. M., Qi, C., \& Dullemond, C. P. 2009, ApJ, 700, 1502 Andrews, Wilner, Hughes, Qi, \& Dullemond, 2010, ApJ, 723, 1241

Andrews, S. M., et al. 2011, ApJ, 732, 42

Beckwith, S. V. W. \& Sargent, A. I. 1991, ApJ, 381, 250

Bergin, E. A., Cleeves, L. I., Gorti, U., et al. 2013, Nature, 493, 644

Calvet, N., et al. 2002, ApJ, 568, 1008

Calvet, N., et al. 2005, ApJL, 630, L185

Espaillat, C., et al. 2007a, ApJL, 664, L111

Espaillat, C., et al. 2008, ApJL, 689, L145

Espaillat, C., et al. 2010, ApJ, 717, 441

Espaillat, C., Furlan, E., D'Alessio, P., et al. 2011, ApJ, 728, 49

Furlan, E., et al. 2006, ApJS, 165, 568

Houck, J. R., et al. 2004, ApJS, 154, 18

Hughes, A. M., et al. 2007, ApJ, 664, 536

Hughes, A. M., et al. 2009, ApJ, 698, 131

Kenyon, S. J. \& Hartmann, L. 1995, ApJS, 101, 117

Luhman, K. L., Allen, P. R., Espaillat, C., Hartmann, L., \& Calvet, N. 2010, ApJS, 186, 111

Paardekooper, S.-J. \& Mellema, G. 2004, A\& A, 425, L9

Skrutskie, M. F., et al. 1990, AJ, 99, 1187

Skrutskie, M. F., et al. 2006, AJ, 131, 1163

Strom, K. M., Strom, S. E., Edwards, S., Cabrit, S., \& Skrutskie, M. F. 1989, AJ, 97, 1451

Thi, W.-F., Mathews, G., Ménard, F., et al. 2010, A\&A, 518, L125

Weaver, W. B. \& Jones, G. 1992, ApJS, 78, 239

Weintraub, D. A., Sandell, G., \& Duncan, W. D. 1989, ApJL, 340, L69

Werner, M. W., et al. 2004, ApJS, 154, 1

Zhu, Z., Nelson, R. P., Dong, R., Espaillat, C., and Hartmann, L. 2012, ApJ, 755, 6

\section{Discussion}

RAFIKOv: Your argument in favour of high dust/gas was that otherwise the gas disk 
mass is $\approx 1 M_{*}$. But that means that initially, in the non-transitional phase, the gas disk still had to be very massive, $\approx 1 M_{*}$. Is this a problem?

ESPAILLAT: If the dust is not accreted or photoevaporated at the same rate as the gas, then the dust per unit mass can increase with time. In this case, the initial gas disk did not need to be much more massive.

LEINHARDT: What is the radial dependence of the gas to dust ratio in transitional disks? Do you expect a significant radial dependence of gas to dust ratio in the outer disk due to dust filtration at the wall?

ESPAILLAT: We currently do not have a good observational constraint on the radial dependence of the gas to dust ratio in any disk. For the transitional disks, if dust filtration is occurring, it is expected that there is an enhancement of dust near the wall and ALMA will be able to test this. 